\title{
A Fully Implicit, Lower Bound, Multi-axial Solution Strategy for Direct Ratchet Boundary Evaluation: Implementation and Comparison
}

\author{
Alan Jappy \\ University of Strathclyde \\ Glasgow, United Kingdom \\ Email: alan.jappy@strath.ac.uk \\ Donald Mackenzie \\ University of Strathclyde \\ Glasgow, United Kingdom \\ Email: d.mackenzie@strath.ac.uk \\ Haofeng Chen \\ University of Strathclyde \\ Glasgow, United Kingdom \\ Email: haofeng.chen@strath.ac.uk
}

\section{ABSTRACT}

Ensuring sufficient safety against ratcheting is a fundamental requirement in pressure vessel design. However, determining the ratchet boundary using a full elastic plastic finite element analysis can be problematic and a number of direct methods have been proposed to overcome difficulties associated with ratchet boundary evaluation. This paper proposes a new lower bound ratchet analysis approach, similar to the previously proposed Hybrid method but based on fully implicit elastic-plastic solution strategies. The method utilizes superimposed elastic stresses and modified radial return integration to converge on the residual state throughout, resulting in one Finite Element model suitable for solving the cyclic stresses (Stage 1) and performing the augmented limit analysis to determine the ratchet boundary (Stage 2). The modified radial return methods for both stages of the analysis are presented, with the corresponding stress update algorithm and resulting consistent tangent moduli. Comparisons with other direct methods for selected benchmark problems are presented. It is shown that the proposed method evaluates a consistent lower bound estimate of the ratchet boundary, which has not previously been clearly demonstrated for other lower bound approaches. Limitations in the description of plastic strains and compatibility during the ratchet analysis are identified as being a cause for the differences between the proposed methods and current upper bound methods. 


\section{NOMENCLATURE}

a Used for calculating $\mathrm{X}$

b Used for calculating $\mathrm{X}$

c Used for calculating $X$

$C_{i j k l} \quad$ Elastic constitutive matrix

E Elastic Modulus

f $\quad$ Yield function

$r_{i j} \quad$ Plastic strain normal directions

$\mathrm{X} \quad$ Used to scale the constant stress for limit analysis

$\delta \quad$ Partial derivative

$\rho_{i j}^{p} \quad$ Residual stress from constant load

$\bar{\rho}_{i j} \quad$ Constant cyclic residual stress

$\rho_{i j}^{r} \quad$ Varying cyclic residual stress

$\hat{\sigma}_{i j} \quad$ Elastic cyclic stress in equilibrium with the cyclic loads at a given point in the load cycle

$\hat{\sigma}_{i j}^{c} \quad$ Elastic constant stress in equilibrium with the constant load

$\sigma_{i j}^{p} \quad$ Stress at loaded state during Stage 2

$\sigma^{y} \quad$ Equivalent yield stress

$\sigma^{y, \bmod }$ Equivalent yield stress used in Stage 2 limit analysis

$\mu \quad$ Shear modulus

$\varepsilon_{i j}^{T} \quad$ Total mechanical strain $\left(\varepsilon_{i j}^{e}+\varepsilon_{i j}^{p}\right)$

$\varepsilon_{i j}^{e} \quad$ Elastic strain

$\varepsilon_{i j}^{p} \quad$ Plastic strain

$\lambda \quad$ Lame's constant

$\gamma \quad$ Equivalent plastic strain

Indices:

$\mathrm{i}, \mathrm{j}, \mathrm{k}, \mathrm{l}$,

$\mathrm{o}, \mathrm{p}, \mathrm{q}, \mathrm{r}$

Possible values are 1,2 ,and 3

Superscripts:

' Deviatoric component

m Cyclic load extreme

$\mathrm{n} \quad$ Increment number 


\section{INTRODUCTION}

An engineering structure subject to a load cycle, in which the loads have differing frequencies and there is at least one primary load, may experience failure through a ratcheting mechanism. This has been demonstrated analytically for a pressurized cylinder by Bree in [1]. For a load cycle with two loads applied at different frequencies, in which the lower frequency load may be considered to be constant,he higher frequency load is referred to as the cyclic load. If the structure does not experience immediate collapse on initial loading, three distinct possible cyclic responses may occur on applying the cyclic load:

1. The initial loading may or may not result in plastic strains in the structure but all subsequent loading results in varying elastic strain only: i.e. the stress everywhere in the structure at every point on the load cycle is less than the yield strength of the material. This response is referred to as elastic shakedown.

2. The initial loading results in plastic strain in the structure and further cyclic loading results in a fully reversed plastic strain cycle in some parts of the structure. This is referred to as plastic shakedown.

3. The initial loading results in plastic strains in the structure and all subsequent cycles result in a net increase in plastic strain in some parts of the structure. The net increase in plastic strain per cycle is referred to as the ratchet mechanism, which can ultimately result in global failure of the component.

Several analysis methods have been proposed for determining or approximating the shakedown boundary, for example [2-6]. Most of these methods predict the boundary between elastic and plastic shakedown with reasonable accuracy, however the ratchet boundary has proved more difficult to establish. In this paper discussion is limited to direct ratchet methods. 'Direct' is used here to mean those methods that are based on augmented limit analysis, however it is recognized that other methods based on linear and non-linear programing methods are available. Of the methods discussed here, upper bound methods, such as the Linear Matching Method [7], have been shown to be effective in calculating the ratchet boundary. The approach presented here is aimed at furthering understanding of the requirements of evaluating lower bounds. The proposed method uses the lower bound conditions given in [8] to develop stress update algorithms, which allow convergence on the cyclic residual stress state, and on an estimate of the constant residual stress state that results at the ratchet boundary due to the application of the constant load. 'Cyclic stresses' are the stabilized stresses which results due to the repeated application of the cyclic loads only and which result in a close strain history. In this method there is no need for simplification in the description of the cyclic loads and an arbitrary cyclic load case may be used, which includes both primary and secondary stresses.

\section{UPPER AND LOWER BOUND RATCHET METHODS}

Direct ratchet boundary methods can be divided into two main groups: lower bound and upper bound methods. Lower bound methods satisfy Melan's lower bound theorem [9], which is concerned with finding a stress conditions that satisfies equilibrium and yield at each point in the load cycle. Upper bound methods establish kinematically admissible strain states that satisfy Koiter's upper bound theorem [10] but do not necessarily satisfy the yield condition. In general, these 
methods calculate an estimate of the cyclic stress condition that is used in an augmented limit analysis, adopting either the upper or lower bound approach. An exception to this is the recently proposed method by Ponter [11] in which the cyclic stresses are not found independently of the constant stresses.

The upper bound Linear Matching method (LMM) and the more recently proposed lower bound non-cyclic method, work in a similar manner. In the LMM, the cyclic response is found by using the method proposed by Chen and Ponter in [12]. The non-cyclic method utilizes the method presented in [13]. Both then use the cyclic stress field in a modulus reduction analysis to find the kinematically admissible strain condition that satisfies Koiter's upper bound theorem [10]. In these analyses, incompressibility in the strain field is maintained through assuming a Poisson's ratio of approximately 0.5. The plastic strains however do not necessarily conform to the normality condition assumed for associative plastic flow. This allows the structure to strain in a manner not consistent with plasticity theory. The modulus reduction technique requires the reduced modulus from a previous iteration. If the method is to be multi-core processed it requires the use of specialist memory types/procedures, or an additional increment to be used to store the reduced modulus in state variable arrays. Consequently, the linear matching method is yet to be multi-core processed.

Current lower bound ratchet methods also include the Hybrid method [14], the lower bound extension to the LMM [15], the Uniform Modified Yield (UMY) and Load Dependent Yield Modification (LDYM) methods [16] and the new methods proposed here. The lower bound extension to the LMM [15] uses the stress fields generated by the upper bound LMM and scales the stresses until the they satisfy the lower bound Melan theorem. Despite using a well-developed upper bound procedure, the lower bound can take a relatively large number of iterations to achieve strict convergence The Hybrid method generates an estimate for the cyclic stresses through the use of the direct cyclic analysis [17] currently available in ABAQUS [18]. The stresses at the limiting points in the cycle are then read into a modified limit analysis [14]. In the Hybrid method the modified yield surface used in the ratchet boundary evaluation is calculated based on initial conditions. This leads to the possibility that the stresses on the ratchet boundary, as calculate by the Hybrid method, could violate Melan's theorem if sufficient redistribution in the stress field occurs, as demonstrated in [14]. Hybrid method ratchet analysis is also subject to many of the same sources of instability and error as the methods presented here. The UMY and LDYM methods [16] use the simplified description of the cyclic stresses proposed by Gokhfeld in [19]. The results for the LDYM method presented in [16] show that the LDYM method can result in ratchet boundaries above that of the upper bound LMM, thus it is currently unclear whether these methods represent a strict lower bound.

The methods proposed here use elastic plastic finite element analysis to converge on the residual cyclic stresses. This model is then used in an augmented limit analysis similar to that proposed by Gokhfeld in [19] and used in [14] and [16]. The modified yield strength is based on the stabilized cyclic stresses [8], as suggested in [20], to determine the ratchet boundary. The use of stabilized cyclic stresses represents a departure from the method proposed by Gokhfled in [19] and used in the non-cyclic method [13] and UMY and LYDM methods [16], as no simplification in the description of the cyclic loads and stresses is applied. The methods presented here are closely linked to the Hybrid method in the use of the stabilized cyclic stresses suggested in [20]. The modified yield strength is updated throughout the analysis, either calculated on the iteration level (method 1) or on the increment level (method 2), both of which account for the 
redistribution in the stress field. Therefore the stresses at the ratchet boundary, as identified by these methods, will satisfy Melan's theorem.

In the methods proposed here and in the Hybrid method, the analysis techniques make use of fully implicit solution methods. This makes them amenable to multi-core processing using standard programing techniques, as results from previous iterations are not required. However despite being able to multi-process the lower bound methods, shorter solution times than the upper bound methods cannot be guaranteed for small numbers of extra cores. This is due to the fact that non-linear analyses require additional iterations to find a stress condition which satisfy equilibrium. Under certain conditions the Hybrid method and the proposed methods can require very small time increments in the solution procedure, further increasing the number of iterations required over the upper bound methods.

\section{PROPOSED LOWER BOUND METHOD}

The proposed method is based on the following assumptions:.

Assumption 1: The load history can be decomposed into constant and cyclic parts.

Assumption 2: $\quad$ The cyclic loads lie within the ratchet boundary.

Assumption 3: The constant load is applied under load control.

Assumption 4: $\quad$ Perfect plasticity is assumed throughout.

Assumption 5: $\quad$ Material properties are temperature independent.

The necessary constitutive models allowing calculation of a stress condition to satisfy the conditions to prevent ratchet and the elastic-plastic constitutive theorems have been developed in a companion paper [8]. Here the stress update algorithms resulting from integration of the plastic strain increment over a load increment in a global Newton-Raphson solution procedure is presented. The global Newton-Raphson solution procedure requires use of a consistent or algorithmic tangent modulus. This gives the linearized form of the rate of change of stress with respect to the total mechanical strain, to conserve the quadratic rate of convergence of the global Newton-Raphson scheme [21]. The consistent tangent moduli for the numerical schemes presented here are given without derivation.

\subsection{NUMERICAL SCHEME}

The proposed method is based on the assumption that the load history can be decomposed into cyclic and constant parts. This allows consideration of the cyclic loads in the absence of constant loads [19].

Stage 1 of the methods considers the cyclic loads only and, if assumption 2 is valid, finds the cyclic stresses which resulting at the shakedown condition for cyclic loads only. Stage 1 does this by performing incremental elastic-plastic analysis for several applications of the cyclic loads only, until the residual stresses and plastic strains converge from one cycle to the next. Here the cyclic loads are assumed to have $m$ extremes that suitably describe the limiting points on the cycle, i.e. those which result in the largest cyclic stresses. 
Indicial notation is adopted, with the indices and their possible values given in the nomenclature section. Where indices are repeated Einstein summation is implied.

\subsection{STAGE 1 NUMERICAL SCHEME}

It is assumed that the material properties are temperature independent but the method is generalized to a multiextreme load cycle. The necessary lower bound, conditions for shakedown are presented in [8] and the required models summarized as:

The stresses at the $m^{\text {th }}$ point on the load cycle are given by:

$\sigma_{i j}^{m}=\bar{\rho}_{i j}+\rho_{i j}^{r m}+\hat{\sigma}_{i j}^{m}$

where $m$ is a point on the load cycle $m=0$ corresponds to the point on the load cycle which is unloaded w.r.t. cyclic loads i.e.:

$\sigma_{i j}^{m}=\bar{\rho}_{i j}$

From the constitutive models formulated in [9], the residual stresses and associated strains must satisfy the condition:

$\bar{\rho}_{i j}+\rho_{i j}^{r m}=C_{i j k l}\left(\varepsilon_{k l}^{m^{m}}-\varepsilon_{k l}^{p m}\right)$

The equivalent von Mises stress is assumed to describe the yielding behavior such that:

$\left|\sigma_{i j}^{\prime m}\right|=\sqrt{\frac{3}{2}\left(\bar{\rho}_{i j}^{\prime}+\rho_{i j}^{r^{\prime} m}+{\hat{\sigma}_{i j}^{\prime}}^{m}\right)\left(\bar{\rho}_{i j}^{\prime}+\rho_{i j}^{r^{\prime} m}+{\hat{\sigma}_{i j}^{\prime}}^{m}\right)}$

with, assuming associative plastic flow at the loaded state:

$\delta \varepsilon_{i j}^{p m}=\delta \gamma r_{i j}^{m}$

$r_{i j}=\frac{\delta f}{\delta \sigma_{i j}}=\frac{3}{2} \frac{{\sigma^{\prime}}^{m}{ }^{m}}{\sqrt{\frac{3}{2}{\sigma^{\prime}}^{\prime}{ }^{m}{ }^{m}{\sigma^{\prime}}_{i j}^{m}}}$

In addition, perfect plasticity is assumed throughout such that $\sigma^{\mathrm{y}}$ is constant, giving the equivalent plastic strain increment:

$\gamma=\frac{r_{i j} C_{i j k l} \varepsilon_{k l}^{T}}{r_{m n} C_{m n o p} r_{o p}}=\frac{r_{i j} C_{i j k l} \varepsilon_{k l}^{T}}{3 \mu}$

The problem then reduces to the choice of integration scheme used to find $\Delta \gamma$ over the increment. As it has been assumed that the plasticity is described by the von Mises yield function and that the flow is associative, it is possible to use J2 plasticity, radial return method to fully implicitly integrate the plastic strain increment. The integration method is defined in detail in [19]. For the case presented here, where the plasticity is assumed to be perfect, the plastic strain increment reduces to: 
$\Delta \gamma^{m}=\frac{f^{\text {trial }^{m}}}{3 \mu}$

The numerical scheme starts at $\mathrm{m}=1$ and on converging on a residual stress condition cycles through the load cycle, using incremental finite element analysis, until the constant residual converges:

$$
\begin{aligned}
& \bar{\rho}_{i j} \quad+\rho_{i j}^{r, \text { trial }^{m}}=C_{i j k l}\left(\varepsilon_{i j}^{T^{m}}-\varepsilon_{i j}^{p^{m-1}}\right) \\
& \varepsilon_{i j}^{T^{\prime} m}=\varepsilon_{i j}^{T}{ }^{m}-\frac{1}{3} \operatorname{tr}\left(\varepsilon_{i j}^{T^{m}}\right) \\
& \varepsilon_{i j}^{e^{\prime}, \text { trial }^{m}}=\varepsilon_{i j}^{T^{\prime} m}-\varepsilon_{i j}^{p m-1} \\
& \left(\bar{\rho}_{i j}^{\prime}+\rho_{i j}^{r^{\prime}, \text { trial }^{m}}\right)=2 \mu\left(\varepsilon_{i j}^{T^{\prime}{ }^{m}}-\varepsilon_{i j}^{p^{m-1}}\right)
\end{aligned}
$$

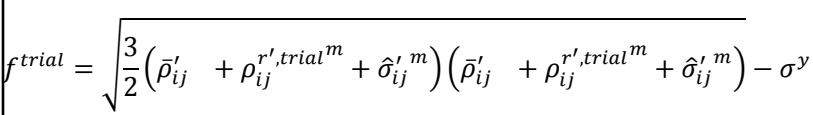

$$
\begin{aligned}
& \text { If } f^{\text {trial }} \leq 0 \text { then: } \\
& \varepsilon_{i j}^{e^{\prime m}}=\varepsilon_{i j}^{e^{\prime}, \text { trial }^{m}} \\
& \varepsilon_{i j}^{p^{m}}=\varepsilon_{i j}^{p^{m-1}} \\
& \bar{\rho}_{i j}^{\prime}+\rho_{i j}^{r^{\prime} m}=\bar{\rho}_{i j}^{\prime}+\rho_{i j}^{r^{\prime}, \text { trial }^{m}} \\
& \bar{\rho}_{i j}+\rho_{i j}^{r m}=\frac{1}{3} \operatorname{tr}\left(\bar{\rho}_{i j}+\rho_{i j}^{r}, \text { trial }^{m}\right)+\left(\bar{\rho}_{i j}^{\prime}+\rho_{i j}^{r^{\prime}, \text { trial }^{m}}\right)
\end{aligned}
$$

Else (plastic):

$$
\begin{aligned}
& r_{i j}^{m}=\frac{3}{2} \frac{\left(\bar{\rho}_{i j}^{\prime}+\rho_{i j}^{r^{\prime}, \text { trial }^{m}}+{\hat{\sigma}_{i j}^{\prime}}^{m}\right)}{f^{\text {trial }}} \\
& \Delta \gamma^{m}=\frac{f^{\text {trial }}}{3 \mu} \\
& \varepsilon_{i j}^{p^{m}}=\varepsilon_{i j}^{p^{m-1}}+\Delta \gamma^{m} r_{i j}^{m} \\
& \left(\bar{\rho}_{i j}^{\prime}+\rho_{i j}^{r^{\prime}}\right)=\left(\bar{\rho}_{i j}^{\prime}+\rho_{i j}^{r^{\prime}, \text { trial }^{m}}\right)-2 \mu \Delta \gamma^{m} r_{i j}{ }^{m} \\
& \bar{\rho}_{i j}+\rho_{i j}^{r m}=\frac{1}{3} \operatorname{tr}\left(\bar{\rho}_{i j}+\rho_{i j}^{r, \text { trial }^{m}}\right)+\left(\bar{\rho}_{i j}^{\prime}+\rho_{i j}^{r^{\prime} m}\right)
\end{aligned}
$$

The consistent tangent modulus is:

$$
C_{i j k l}^{c o n}=\kappa 1_{i j} 1_{k l}+2 \theta \mu\left(I_{i j k l}-\frac{1}{3} 1_{i j} 1_{k l}\right)-\frac{4}{3} \mu \varphi r_{i j}{ }^{m} r_{k l}^{m}
$$

where:

$\mu=\frac{E}{2(1+v)}, \quad \kappa=\frac{E}{3(1-2 v)}, \quad \theta=\frac{\sigma^{y}}{\left|\rho_{o p}^{c, \text { trial }^{n}}\right|}$ and $\varphi=\theta$

Endif 
If $m=0$ then check convergence on $\bar{\rho}_{i j}^{\prime}$

if $\rho_{i j}^{r^{\prime 0}} \leq$ tolerance Goto stage 2

else $\bar{\rho}_{i j}=\bar{\rho}_{i j}+\rho_{i j}^{r}$

Endif

If stress state converges $m=m+1$, go to next global increment

\section{BOX 1: STAGE 1 ALGORITHIM}

\subsection{STAGE 2 NUMERICALSCHEME}

After the stabilized cycle has been found during Stage 1, the magnitude of constant load which can act in addition to the cyclic loads without causing the structure to ratchet is established.

The necessary constitutive model which determines the magnitude of constant load, that can be added to the structure without causing ratchet is formulated in [8] and summarized below, in terms of the residual stress:

$\rho_{i j}^{p}=C_{i j k l}\left(\varepsilon_{k l}^{T}-\varepsilon_{k l}^{p}\right)$

The equivalent von Mises stress is assumed to describe the yielding behavior such that, with superimposed elastic stresses:

$\left|\sigma_{i j}^{p^{\prime}}\right|=\sqrt{\frac{3}{2} \sigma_{i j}^{p^{\prime} \sigma_{i j}^{p^{\prime}}}}=\sqrt{\frac{3}{2}\left(\rho_{i j}^{p^{\prime}}+\hat{\sigma}_{i j}^{c^{\prime}}\right)\left(\rho_{i j}^{p^{\prime}}+\hat{\sigma}_{i j}^{c \prime}\right)}$

The yield stress is modified according to:

$\sigma^{y, \text { mod }}=\sqrt{\frac{3}{2}\left(X \sigma_{i j}^{P^{\prime}}\right)\left(X \sigma_{i j}^{P^{\prime}}\right)}=X \sqrt{\frac{3}{2} \sigma_{i j}^{P^{\prime}} \sigma_{i j}^{P \prime}}$

where $\mathrm{X}$ is given by the smallest for all $\mathrm{m}$ of:

$X^{m}=\frac{-b^{m}+\sqrt{b^{m^{2}}-4 a c^{m}}}{2 a}$

where:

$a=\frac{3}{2} \sigma_{i j}^{P^{\prime}} \sigma_{i j}^{P^{\prime}} \quad b^{m}=3 \sigma_{i j}^{P^{\prime}} \sigma_{i j}^{m \prime} \quad$ and $c^{m}=\frac{3}{2} \sigma_{i j}^{m^{\prime}} \sigma_{i j}^{m \prime}-\left(\sigma^{y}\right)^{2}$

where $\sigma_{i j}^{m}$ are taken from the converged solution at the end of Stage 1.

Assuming, associative plastic flow:

$r_{i j}=\frac{\delta f}{\delta \sigma_{i j}^{p}}=\frac{3}{2} \frac{\sigma_{i j}^{p \prime}}{\sqrt{\sigma_{i j}^{p \prime} \sigma_{i j}^{p \prime}}}$

Giving: 
$\gamma=\frac{r_{i j} C_{i j k l} \varepsilon_{k l}^{T}}{r_{q r} C_{q r o p} r_{o p}}=\frac{r_{i j} C_{i j k l} \varepsilon_{k l}^{T}}{3 \mu}$

As with Stage 1, the model may be integrated with the $\mathrm{J} 2$ radial return method. The numerical scheme then becomes:

$\rho_{i j}^{c, \text { trial }^{n}}=C_{i j k l}\left(\varepsilon_{i j}^{T^{n}}-\varepsilon_{i j}^{p^{n-1}}\right)$

$\varepsilon_{i j}^{T, n}=\varepsilon_{i j}^{T}{ }^{n}-\frac{1}{3} \operatorname{tr}\left(\varepsilon_{i j}^{T}{ }^{n}\right)$

$\varepsilon_{i j}^{e^{\prime}, \text { trial }}=\varepsilon_{i j}^{T{ }^{\prime} n}-\varepsilon_{i j}^{p^{n-1}}$

$\rho_{i j}^{p^{\prime} . \text { trial }}=2 \mu\left(\varepsilon_{i j}^{T{ }^{\prime} m}-\varepsilon_{i j}^{p^{\prime m-1}}\right)$

$\mathrm{X}$ is the smallest for all $\mathrm{m}$ of:

$X^{m}=\frac{-b^{m}+\sqrt{b^{m^{2}}-4 a c^{m}}}{2 a}$

Where:

$a=\frac{3}{2} \sigma_{i j}^{P \prime} \sigma_{i j}^{P \prime} \quad b^{m}=3 \sigma_{i j}^{P \prime} \sigma_{i j}^{m \prime} \quad$ and $\quad c^{m}=\frac{3}{2} \sigma_{i j}^{m \prime} \sigma_{i j}^{m \prime}-\left(\sigma^{y}\right)^{2}$

$\sigma^{y, \text { mod }}=\sqrt{\frac{3}{2}\left(X \sigma_{i j}^{P^{\prime}, \text { trial }}\right)\left(X \sigma_{i j}^{P^{\prime}, \text { trial }}\right)}=X \sqrt{\frac{3}{2}\left(\sigma_{i j}^{P^{\prime}, \text { trial }}\right)\left(\sigma_{i j}^{P^{\prime}, \text { trial }}\right)}$

$f^{\text {trial }}=\sqrt{\frac{3}{2}\left(\rho_{i j}^{\text {p',trial },}+\hat{\sigma}_{i j}^{c \prime \prime}\right)\left(\rho_{i j}^{\text {p',trial }}+\hat{\sigma}_{i j}^{c \prime n}\right)}-\sigma^{y \cdot m o d}$

If $f^{\text {trial }} \leq 0$ then:

$\varepsilon_{i j}^{e^{\prime n}}=\varepsilon_{i j}^{e^{\prime}, \text { trial }^{n}}$

$\varepsilon_{i j}^{p^{n}}=\varepsilon_{i j}^{p n-1}$

$\rho_{i j}^{p^{\prime \prime}}=\rho_{i j}^{p^{\prime n-1}}$

$\rho_{i j}^{p^{n}}=\frac{1}{3} \operatorname{tr}\left(\rho_{i j}^{p, \text { trial }^{n}}\right)+\left(\rho_{i j}^{p^{\prime n}}\right)$

Else (plastic):

$r_{i j}^{n}=\frac{3}{2} \frac{\rho_{i j}^{p^{\prime}, \text { trial }}+\hat{\sigma}_{i j}^{c \prime n}}{f^{\text {trial }}}$ 


$$
\left\{\begin{array}{l}
\Delta \gamma^{n}=\frac{f^{\text {trial }}}{3 \mu} \\
\varepsilon_{i j}^{p^{n}}=\varepsilon_{i j}^{p^{n-1}}+\Delta \gamma \quad r_{i j}^{n} \\
\rho_{i j}^{p^{\prime} n}=\rho_{i j}^{p^{\prime}, \text { trial }}-2 \mu \Delta \gamma \quad r_{i j}^{n} \\
\rho_{i j}^{p^{n}}=\frac{1}{3} \operatorname{tr}\left(\rho_{i j}^{p, \text { trial }}{ }^{n}\right)+\rho_{i j}^{p^{\prime}}{ }^{n}
\end{array}\right.
$$

The consistent tangent modulus is then:

$$
\begin{gathered}
C_{i j k l}^{c o n}=\kappa 1_{i j} 1_{k l}+2 \theta \mu\left(I_{i j k l}-\frac{1}{3} 1_{i j} 1_{k l}\right)-\frac{4 \mu}{3}\left(2 X+2 \frac{c}{\sqrt{b^{m^{2}}-4 a c^{m}}}\right) r_{i j} r_{k l} \\
-4 \mu \frac{X\left|\sigma_{o p}^{P^{\prime}, \text { trial }}\right|}{\sqrt{b^{m^{2}}-4 a c^{m}}} r_{i j}\left(\bar{\rho}_{k l}^{\prime}+\rho_{k l}^{r^{\prime}}+\hat{\sigma}_{k l}^{\prime m}\right)
\end{gathered}
$$

where the superscript $m$ corresponds to the point on the load cyclic which gave the limiting case of X:

$\mu=\frac{E}{2(1+v)}, \quad \kappa=\frac{E}{3(1-2 v)}, \quad \theta=\frac{\sigma^{y, \text { mod }}}{\left|\rho_{o p}^{p, t r i a l^{n}}+\widehat{\sigma}_{o p^{\prime}}^{c}\right|} \mid=X$

Endif

\section{BOX 2: METHOD 1, STAGE 2 NUMERICAL SCHEME}

\section{COMMENTS ON NUMERICAL SCHEMES}

The numerical schemes are both based on the radial return method, which gives a relatively simple integration of the plastic strain increment and results in closed form consistent tangent moduli. However, this approach has a number of associated problems. The integration of the plastic strain increment assumes a constant normal direction throughout the increment. In situations where there is stress redistribution, which results in a change to the stress normals, this produces a relatively good approximation. However, if there is redistribution in which the stress normal directions change throughout the increment, this could result in an error in the plastic strains as the 'true' history of the plastic strains is not captured. If accuracy in the plastic strain directions is required then, in cases where there is redistribution in the stress state, the magnitude of the plastic strain increment must be limited.

Analysis of the consistent tangent modulus developed for Stage 1shows that if the term $\theta=\frac{\sigma^{\mathrm{y}}}{\left|\rho_{\mathrm{op}}^{\mathrm{c}, \text { trial }^{\mathrm{n}}}\right|}$ becomes small then the consistent tangent modulus can become ill conditioned [21]. In such cases the global Newton-Raphson solver will fail to converge. To overcome this problem the plastic strain increment is limited to ensure $\theta=\frac{\sigma^{y}}{\left|\rho_{o p}^{c, t r i a l} l^{n}\right|}$ is large enough to maintain stability in the solution. In the examples presented in Section 6 the term $\theta=\frac{\sigma^{y}}{\mid \rho_{\text {op }}^{\text {ctrial }}{ }^{n}} \mid$ was kept above 0.25 . 
This may require additional points in the load cycle to be used in addition to the limiting extremes. For example, if the

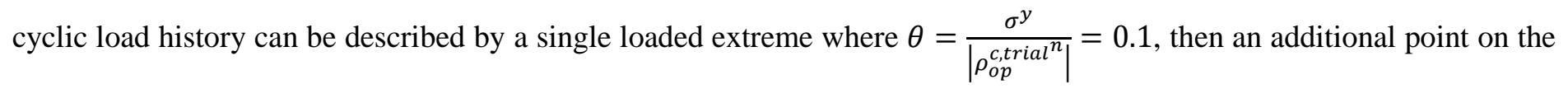
load cycle must be identified where $\theta=\frac{\sigma^{y}}{\mid \rho_{o p}^{c, t r i a l} l^{n}} \geq 0.25$. Stage 1 is then conducted using those two points as cyclic load extremes, whichdoes not affect the final solution and allows convergence of the global Newton-Raphson solution procedure. This particular problem arises due to the use of fixed time stepping during Stage 1 and could be eliminated by developing a Stage 1 procedure that makes use of automatic time stepping.

The consistent tangent modulus of Stage 2 exhibits similar behavior, despite using automatic time stepping, with $\theta=\frac{\sigma^{\mathrm{y}, \text { mod }}}{\left|\rho_{\text {op }}^{\text {c.trial }}{ }^{\mathrm{n}}\right|}=\mathrm{X} \quad$ but in this case the problem can be more pronounced as $\sigma^{\mathrm{y}, \mathrm{mod}} \rightarrow 0$. This might require use of relatively small increments during the Stage 2 limit analysis. The increment size can be increased by limiting the lower value of $\sigma^{\mathrm{y} \text {,mod }}$. In the examples presented in Section 6 , the lower limit on $\sigma^{\mathrm{y}, \text { mod }}$ is $\sigma^{\mathrm{y}, \bmod } \geq 0.01 \mathrm{MPa}$. By limiting the lower bound of $\sigma^{\mathrm{y}, \mathrm{mod}}$, a slight over-estimation of the lower bound solution occurs, however if the volume of the structure at which this limit has been applied is small compared to the failure plane, the resulting error can be assumed to be negligible.

The consistent tangent modulus derived for Stage 2 can become ill conditioned under certain conditions of stress. In the examples presented in Section 6 the consistent tangent modulus maintained the rate of convergence up to a point at which a sudden decrease in solution rate was observed, see fig. 5, fig. 6 and fig. 7. In such cases the yield stress calculated in the previous increment can be assumed to be constant through the current increment, see Section 5. This may result in a local violation of Melan's theorem, if the modified yield strength reduces during the increment as a result of redistribution. If however the final increment in the analysis is small, $\sigma^{\mathrm{y}, \bmod ^{\mathrm{n}}} \approx \sigma^{\mathrm{y}, \bmod ^{\mathrm{n}-1}}$, any violation of Melan's theorem can be assumed negligible and the solution may be assumed to be a lower bound.

\section{CHANGE TO STAGE 2 NUMERICAL SCHEME}

If the consistent tangent modulus derived for Stage 2 becomes ill conditioned, the solver may fail due to poor convergence rate or the solution requiring increments too small to solve. To avoid this, the Stage 2 scheme can be modified to give a more reliable solution of the ratchet boundary. The modified scheme is designed to avoid instability by using the yield strength calculated at the start of the increment and changing the consistent tangent modulus appropriately. This integration scheme is referred to as Method 2

$$
\begin{aligned}
& \rho_{i j}^{p, \text { trial }^{n}}=C_{i j k l}\left(\varepsilon_{i j}^{T^{n}}-\varepsilon_{i j}^{p^{n-1}}\right) \\
& \varepsilon_{i j}^{T{ }^{\prime}}{ }^{n}=\varepsilon_{i j}^{T^{n}}-\frac{1}{3} \operatorname{tr}\left(\varepsilon_{i j}^{T^{n}}\right) \\
& \varepsilon_{i j}^{e^{\prime}, \text { trial }}=\varepsilon_{i j}^{T{ }^{\prime}}{ }^{n}-\varepsilon_{i j}^{p^{n-1}}
\end{aligned}
$$


$\rho_{i j}^{p^{\prime} . \text { trial }}=2 \mu\left(\varepsilon_{i j}^{T{ }^{\prime m}}-\varepsilon_{i j}^{p^{\prime m-1}}\right)$

$\mathrm{X}$ is the smallest for all $m$ of:

$X^{m}=\frac{-b^{m}+\sqrt{b^{m^{2}}-4 a c^{m}}}{2 a}$

Where:

$a=\frac{3}{2} \sigma_{i j}^{P \prime} \sigma_{i j}^{P \prime} \quad b^{m}=3 \sigma_{i j}^{P \prime} \sigma_{i j}^{m \prime} \quad$ and $\quad c^{m}=\frac{3}{2} \sigma_{i j}^{m \prime} \sigma_{i j}^{m \prime}-\left(\sigma^{y}\right)^{2}$

$\sigma^{y, \text { mod }^{n}}=\sqrt{\frac{3}{2}\left(X \sigma_{i j}^{P^{\prime}, \text { trial }}\right)\left(X \sigma_{i j}^{P^{\prime}, \text { trial }}\right)}=X \sqrt{\frac{3}{2}\left(\sigma_{i j}^{P^{\prime}, \text { trial }}\right)\left(\sigma_{i j}^{P^{\prime}, \text { trial }}\right)}$

$f^{\text {trial }}=\sqrt{\frac{3}{2}\left(\rho_{i j}^{p^{\prime}, \text { trial }}+\hat{\sigma}_{i j}^{c}{ }^{\prime} n\right)\left(\rho_{i j}^{p^{\prime}, \text { trial }}+\hat{\sigma}_{i j}^{c \prime n}\right)}-\sigma^{y \cdot m o d^{n-1}}=$

If $\mathrm{f}^{\text {trial }} \leq 0$ then:

$\varepsilon_{i j}^{e^{\prime n}}=\varepsilon_{i j}^{e^{\prime}, \text { trial }^{n}}$

$\varepsilon_{i j}^{p^{n}}=\varepsilon_{i j}^{p n-1}$

$\rho_{i j}^{p^{\prime n}}=\rho_{i j}^{p^{\prime n-1}}$

$\rho_{i j}^{c}{ }^{n}=\frac{1}{3} \operatorname{tr}\left(\rho_{i j}^{p, \text { trial }^{n}}\right)+\left(\rho_{i j}^{p^{\prime n}}\right)$

Else if $f^{\text {trial }}>0$ (plastic):

$r_{i j}^{n}=\frac{3}{2} \frac{\rho_{i j}^{p^{\prime}, \text { trial }}+\hat{\sigma}_{i j}^{c \prime n}}{f^{\text {trial }}}$

$\Delta \gamma^{n}=\frac{f^{\text {trial }}}{3 \mu}$

$\varepsilon_{i j}^{p n}=\varepsilon_{i j}^{p^{n-1}}+\Delta \gamma \quad r_{i j}^{n}$

$\rho_{i j}^{p^{\prime n}}=\rho_{i j}^{p^{\prime, \text { trial }}}-2 \mu \Delta \gamma \quad r_{i j}^{n}$

$\rho_{i j}^{p^{n}}=\frac{1}{3} \operatorname{tr}\left(\rho_{i j}^{p, \text { trial }^{n}}\right)+\rho_{i j}^{p^{\prime}}$

The consistent tangent modulus is then:

$C_{i j k l}^{c o n}=\kappa 1_{i j} 1_{k l}+2 \theta \mu\left(I_{i j k l}-\frac{1}{3} 1_{i j} 1_{k l}\right)-\frac{4}{3} \mu \theta r_{i j}{ }^{m} r_{k l}{ }^{m}$ 
$\mu=\frac{E}{2(1+v)}, \kappa=\frac{E}{3(1-2 v)}, \theta=\frac{\sigma^{y, m o d^{m-1}}}{\left|\rho_{o p}^{p, \text { trial }^{n}}+\widehat{\sigma}_{o p^{\prime}}^{c}{ }^{n}\right|}$

Endif

BOX 3: METHOD 2, STAGE 2 NUMERICAL SCHEME

\section{NUMERICAL APPLICATIONS}

The stress update algorithms have been implemented in ABAQUS using the UMAT subroutine, although it is recognized that other Finite element codes could also be used. The method developed in Section 3 is referred to as Method 1 , and the method given in Section 5 is referred to as Method 2.

\subsection{BREE CYLINDER}

The Bree cylinder is a common bench mark for new direct shakedown and ratchet methods as it presents a case which can be analyzed analytically. Here the axisymmetric case considered in [14] is used to investigate application of the proposed methods. The hybrid solutions were obtained using a variation of the model proposed here and show good agreement with the original solutions in [14], verifying the use of the residual stress state during the analysis. Note that little redistribution of stress occurs in the Bree cylinder so little difference is expected between the proposed method and the Hybrid method.

The Finite Element model dimensions are given in fig. 1. The model was created using second order reduced integration axi-symmetric elements, with 10 elements of aspect ratio 1 through thickness. The material properties were Young's modulus of $184 \mathrm{GPa}$, Poison's ratio of 0.3 and yield strength $402.7 \mathrm{MPa}$. The cylinder is subject to a constant internal pressure and corresponding axial thrust simulating end cap pressure. The cyclic load is applied through a cyclic internal temperature: the external temperature is fixed at all times to $20^{\circ} \mathrm{C}$, the temperature gradient is found by thermal analysis with thermal conductivity of $0.035 \mathrm{~W} / \mathrm{mm}$ and the resultant cyclic stress is found with a thermal expansion coefficient of $1.335 \mathrm{e}-5^{\circ} \mathrm{C}^{-1}$. The end of the cylinder that has the end cap thrust applied is constrained to remain in plain section.

The results given in fig. 2 show that both of the proposed methods produce nominally the same results as the hybrid method due to limited amount of redistribution in the stress field. The results also show that the proposed methods do not overcome the deviation of the calculated boundary from the linear matching method. This is not due to the method being a lower bound, as fig. 2 shows that the lower bound extension to the LMM can determine a lower bound closer to that given by the upper bound LMM.

The resulst presented in fig 2 indicate that there may be a fundamental limitation to the vector summation type of direct ratchet methods such as the hybrid method and the methods presented here. In the Bree cylinder analysis, all of the methods were able to find the ratchet condition; that is, the equivalent stress in the cylinder is equal to yield at some point on the load cycle thus there is no further potential for the structure to hold additional constant load. This is shown in fig. 3 , 
which gives the equivalent stress in the structure for $\Delta \theta=500$ at the $\theta=20$ and $\theta=520$ points on the load cycle. It is seen that the inner portion of the cylinder is limited by the $\theta=20$ load case and the outer portion of the cylinder is limited by the $\theta=520$ load case. This indicates that no further improvement can be achieved on the ratchet boundary with the current vector type lower bound methods, and the approach must be fundamentally changed to achieve better agreement with the LMM.

\subsection{PRESSURISED TWO BAR STRUCTURE}

The pressurized bar structure shown below in fig. 4, modified from [22] and analyzed in [14], is investigated here to obtain further insight into the deviation of the ratchet boundary prediction from that of the linear matching method. . Bar 1 has an internal radius of $2.00 \mathrm{~mm}$ and an external radius of $2.68 \mathrm{~mm}$. Bar 2 has an internal radius of $2.00 \mathrm{~mm}$ and an external radius of $3.22 \mathrm{~mm}$. Bar 2 is twice the length of bar 1 and both have the same material properties: Young's modulus of $210 \mathrm{GPa}$, Poisson's ratio of 0.3 , yield strength of $200 \mathrm{MPa}$ and a thermal expansion coefficient of $1.17 \times 10^{-5^{\circ}} \mathrm{C}^{-}$

1. The ends of the cylinders are constrained to remain in plane section at all times. The model was defined in 3D with second order reduced integration elements, with six elements through the thickness of bar 2 and 3 through the thickness of bar 1.

The load cycle is described by a constant load which consists of internal pressure applied to bar 2 without a corresponding end cap pressure and an axial force which is distributed between both bars by a plane section constraint. The cyclic load is applied as a varying temperature, above the reference temperature, in bar 2, with the temperature being uniform throughout the bar. Bar 1 remains at the reference temperature at all times. Pressure and axial force are considered for three separate conditions $\quad:$ where the force $F$ in Newtons divided by the pressure $P$ in $M P a$ is $F / P=10$, 15 and 20. This is done to test the proposed method under varying extents of multi-axial stress conditions. The ratchet boundary as determined by the proposed method, the hybrid method and the LMM are given in figs. 5 to 7.

The results show that the proposed method produces different results to the Hybrid method for the varying degrees of multi-axial stress condition. In most of the points considered Method 1 produces results below that of the hybrid method. This can be attributed to the consistent tangent modulus becoming ill conditioned, resulting in a premature failure of the ratchet solution. Method 2 produces a boundary more consistent with the upper bound linear matching method in all of the conditions analyzed. Both of the proposed methods avoid the possibility of the non-conservative results seen in the hybrid method. For $F / P=20$ at a cyclic temperature above approximately $140^{\circ} \mathrm{C}$ this can be attributed to the hybrid method not considering the effective weakening of the structure caused by stress redistribution, see fig. 8 .

\section{DISCUSSION}

The proposed lower bound direct ratchet method solution schemes account for stress redistribution through modification of the yield stress when the constant load is applied. The method is therefore able to account for increased strength when the stress redistribution is beneficial and reduced strength when the stress distribution is detrimental, leading to strict lower bound solutions in both cases. Fig 8 shows the modified yield stress in the pressurized two bar 
example components at load $\mathrm{F} / \mathrm{P}=20$ and $\Delta \theta=150^{\circ} \mathrm{C}$ evaluated by the Hybrid method and the proposed method. The reduced yield strength shown for the proposed method ensures that the calculated ratchet boundary is conservative, whereas the Hybrid method does not simulate this response.

Two plastic strain increment integration strategies have been implemented in the method. Both give good agreement with the hybrid method for the Bree cylinder, due to the limited stress redistribution occurring in that example. When more extensive stress redistribution occurs in problems such as the plate with hole in [8] and the pressurized two bar problem, numerical instability can occur during Stage 2 limit analysis of Method 1, resulting in premature termination of the analysis. This instability may be due to competing limiting stress conditions within the solution. In the Bree cylinder example, a single load case limits the response at each point in the structure and a consistent cyclic load defines the consistent tangent modulus, leading to a relatively stable solution. However, in the pressurized two bar problem the structure can develop a stress field in which more than one extreme point in the load cycle must be limited to the yield stress. Fig 9 shows the total equivalent stress in the two-bar structure members at $\mathrm{F} / \mathrm{P}=15$ with $\Delta \theta=0^{\circ} \mathrm{C}$ and $\Delta \theta=150^{\circ} \mathrm{C}$. In the latter case, the stress distribution could result in a rapidly changing modulus causing instability. Other sources of instability could also contribute to premature solver failure. Method 2 addresses this problem by updating the modified yield surface using converged solutions from the previous increment, resulting in a relatively stable solution method. However, Method 2 still demonstrates considerable deviation from the upper bound ratchet method solutions. Therefore, whilst the ratchet boundary given by Methods 1 and 2 will be conservative it could be excessively conservative under multi-axial stress conditions.

The example problems considered show that the proposed lower bound methods are in limited agreement with LMM upper bound solutions. However, the lower bound approximation of the LMM demonstrates that it should be possible to establish residual stress fields closer to the upper bound solution that satisfy Melan's theorem. Possible reasons for the discrepancies in the proposed method are compatibility errors in the solution procedure and inconsistent evaluation of plastic strain direction..

The stress distribution in the pressurized bar problem at $\mathrm{F} / \mathrm{P}=20$ is predominantly uniaxial and the Method 2 results show reasonable agreement with the upper bound LMM except at the point at $\Delta \theta=50^{\circ} \mathrm{C}$. The cyclic temperature produces a stress in bar 1 causing yield at cyclic temperature of approximately $84^{\circ} \mathrm{C}$ and reverse plasticity at $168^{\circ} \mathrm{C}$. Beyond $168^{\circ} \mathrm{C}$, bar 1 becomes perfectly flexible to additional strain caused by the thermal load in bar 2 . Above $168^{\circ} \mathrm{C}$ the Method 2 results are independent of increasing temperature and exhibit good agreement with the LMM solution (this behavior is also observed for $\mathrm{F} / \mathrm{p}=10$ and 15 ). Below $168^{\circ} \mathrm{C}$, the difference between the Method 2 and LMM solutions may be due to incompatibility between the two bars and within individual bars.

The Method 2 Bree cylinder results are different to the LMM for cyclic temperatures considerably below the first yield and reverse plasticity loads. At these points compatibility issues caused by cyclic solutions should not occur. However, the plane section constraint applied to the free end of the cylinder may significantly influence the local stress state resulting from a given plastic strain direction. The plastic strain which would result in the actual structure would be that from the combination of constant plus cyclic load, thus the normal directions would be: 


$$
r_{i j}{ }^{n}=\frac{3}{2} \frac{\rho_{i j}^{p^{\prime}, \text { trial }}+\widehat{\sigma}_{i j}^{\mathrm{p} / n}+\sigma_{i j}^{\prime}{ }^{m}}{\left|\rho_{i j}^{p^{\prime}, \text { trial }}+\widehat{\sigma}_{i j}^{p / n}+\sigma_{i j}^{\prime}{ }^{\mathrm{m}}\right|}
$$

The difference in normal directions may have a significant impact on the axial strain in the parts of the structure that yield, which would then affect the plane section constraint and the resulting redistribution of stress. A similar situation may occur in bar 2 of the pressurized two bar structure, giving a possible reason for the greater deviation of the results in the elastic shakedown region for $F / P=10$ and $F / P=15$.

Further work is required to better understand the effect of compatibility and plastic strain directions during the Stage 2 limit analysis. Whilst carrying over the plastic strains from Stage 1 is relatively simple, using the actual plastic strain direction would make identifying a limiting case more difficult as the direction of plastic strain would be different for different cyclic loads.

\section{CONCLUSIONS}

The proposed lower bound direct ratchet methods account for stress in a cyclically loaded structure when a constant load is applied. This approach achieves closer agreement with the LMM upper bound solution than the lower bound Hybrid method. This feature also removes the possibility of evaluating a non-conservative estimate of the ratchet boundary.

The example problems presented identify two possible limitations on vector summation type lower bound ratchet methods: loss of plastic strains between the cyclic and ratchet stages of the analysis resulting in compatibility problems and the calculation of the plastic strain direction based on the constant load only.

To increase the reliability of the proposed lower bound ratchet methods, further work is required to fully understand the impact of compatibility and plastic strain directions during Stage 2 of the solution procedure.

\section{ACKNOWLEDGEMENTS}

The authors would like to acknowledge the contributions of Mr James Ure, University of Strathclyde Glasgow for his lower bound LMM results and also the contributions of Mr Michael Lytwyn, University of Strathclyde Glasgow for his upper bound LMM results.

\section{REFERENCES}

[1] Bree, J., 1967, "Elasto-plastic behaviour of thin tubes subjected to internal pressure and intermittent heat fluxes with application to fast reactor fuel elements," J. Strain. Analysis, 2, pp.226-238.

[2] Chen, H.F., 2010, "Lower and Upper Bound Shakedown Analysis of structures With Temperature-Dependent Yield Stress,” Journal of Pressure Vessel Technology, pp.132, 1-8

[3] Staat, M., Heitzer, M., 2001, "LISA a European Project for FEM-based Limit and Shakedown Analysis," Nuclear Engineering and Design 206, pp.151-166. 
[4] Muscat, M., Mackenzie, D. and Hamilton, R., 2003, "Evaluating shakedown by non-linear static analysis," Computers and Structures, 81, pp.1727-1737.

[5] Abdalla, H.F., Megahed, M.M., Younan, M.Y.A., 2007, "A Simplified Technique for Shakedown Limit Load Determination," Nuclear Engineering and Design, 237, pp.1231-1240.

[6] Mackenzie D, Boyle J T, Hamilton R., 2000, “The Elastic Compensation Method for Limit and Shakedown Analysis: a Review", The J. Strain Analysis for Engineering Design, 35 (3), pp.171-188.

[7] Chen, H.F., 2010, "A Direct Method on the Evaluation of Ratchet Limit," J. Pressure Vessel Technology, 132, 041202

[8] Jappy, A., Mackenzie, D., Chen, H., 2012, “A Fully Implicit, Lower Bound, Multi-axial Solution Strategy for Direct Ratchet Boundary Evaluation: Theoretical Development," PVP2012-78314, July 15-19, Toronto, Ontario, CANADA

[9] Melan, E., 1936, “Theorie Statisch Unbestimmter Systeme aus Ideal-Plastischem Bastoff," Sitzungsberichte der Akademie der Wissenschaft. Wien, Abtiia, 145, pp.195-218.

[10] Koiter, W.T., 1960, “General theorems for elastic plastic solids," Progress in solid mechanics, J.N. Sneddon and R. Hill, eds. North Holland, Amsterdam, 1, pp.167-221.

[11] Weichert, D., Ponter A., 2009, Limit States of Materials and Structures, Springer Science+Business Media

[12] Chen, H., Ponter, A., 2001, "A method for the evaluation of a ratchet limit and the amplitude of plastic strain for bodies subjected to cyclic loading,” Eur. J. Mech. A/Solilds, 20, pp.555-571.

[13] Adibi-Asi, R., Reinhardt, W., 2010, Ratchet Boundary Determination Using a Noncyclic Method. Trans. ASME, J. Pressure Vessel Technology; 132, 021201-021201-9

[14]Martin, M., 2009, “A hybrid procedure for ratchet boundary prediction,” Pressure Vessels and Piping, Prague, Czech Republic

[15] Ure, J., Chen, H., Li, T., Chen, W., Tipping, D., Mackenzie, D., 2011, “A Direct Method for the Evaluation of Lower and Upper Bound Ratchet Limits," International Conference on the Mechanical Behaviour of Materials. June 5th-9th, Lake Como, Italy

[16] Abou-Hanna, J., McGreevy, T. E., 2011, “A simplified ratchet limit analysis using modified yield surface,” Int. J. of Pressure Vessel and Piping, 88, 11-18

[17]Nguyen-Tajan, et al. 2003, "Determination of the stabilized response of a structure undergoing cyclic thermalmechanical loads by a direct cyclic method," Abaqus Users' Conference Proceedings

[18] 2010, Abaqus 6.10, SIMULIA

[19] Gokhfeld, D.A. and Cherniavsky , O.F., 1980, Limit Analysis of Structures at Thermal Cycling, Sijthoff \& Noordhoff

[20] Polizzotto, C., 1993, “A Study on Plastic Shakedown of Structures: Part II- Theorems,” Trans. ASME, J. Applied Mechanics, 60, pp.20-25.

[21] Simo, J. C, Hughes, T.J.R, 2000, Computational Inelasticity, Springer-Verlag

[22] Abdalla, H.F, Megahed, M.M., Younan, M.Y.A., 2007, “A Simplified Technique for Shakedown Limit Load Determination," Nuclear Engineering and Design, 237, pp.1231-1240. 


\section{List of Figures}

FIG. 1: BREE CYLINDER WITH CAPPED END: DIMENSIONS

FIG. 2: RATCHET BOUNDARY: BREE

FIG. 3: TOTAL EQUIVALENT STRESS $\Delta \theta=500$ :LEFT $\theta=20$, RIGHT $\theta=520$

FIG. 4: CONSTRAINTS FOR PRESSURIZED TWO BAR STRUCTURE

FIG. 5: RATCHET BOUNDARY F/P=10

FIG. 6: RACHET BOUNDARY F/P=15

FIG. 7: RATCHET BOUNDARY F/P=20

FIG. 8: MODIFIED YIELD STRENGTH F/P=20 $\Delta \theta=150^{\circ} \mathrm{C}$ LEFT: HYBRID, RIGHT: PROPOSED METHODS

FIG. 9: EQUIVALENT TOTAL STRESS $\mathrm{f} / \mathrm{p}=15$ Left: $\Delta \theta=0^{\circ} \mathrm{C}$, Right: $\Delta \theta=150^{\circ} \mathrm{C}$ 


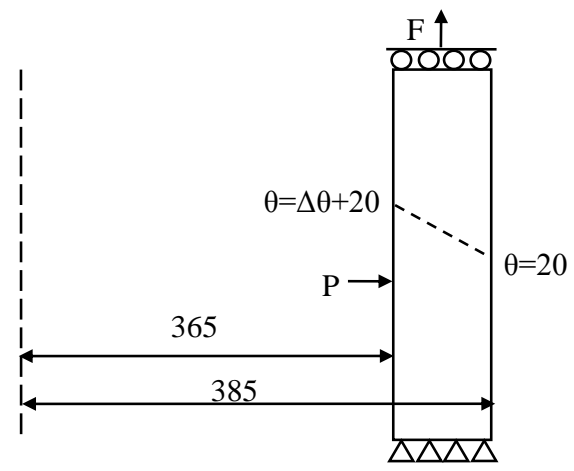

FIG. 1: BREE CYLINDER WITH CAPPED END: DIMENSIONS 


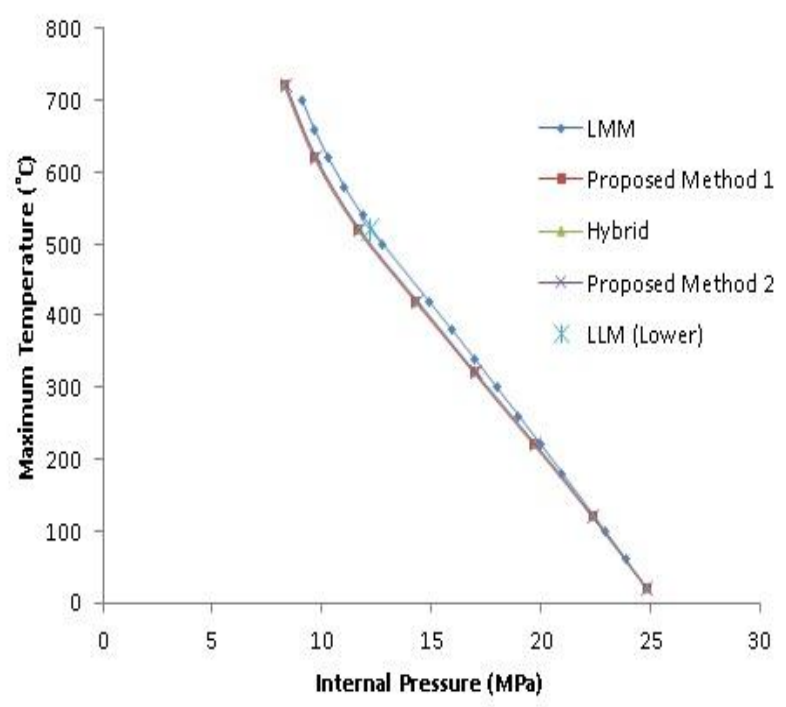

FIG. 2: RATCHET BOUNDARY: BREE 


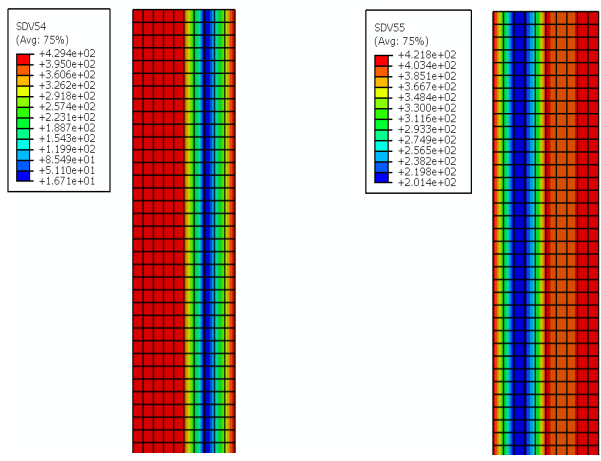

FIG. 3: TOTAL EQUIVALENT STRESS $\Delta \theta=500$ : LEFT $\theta=20$, RIGHT $\theta=520$ 


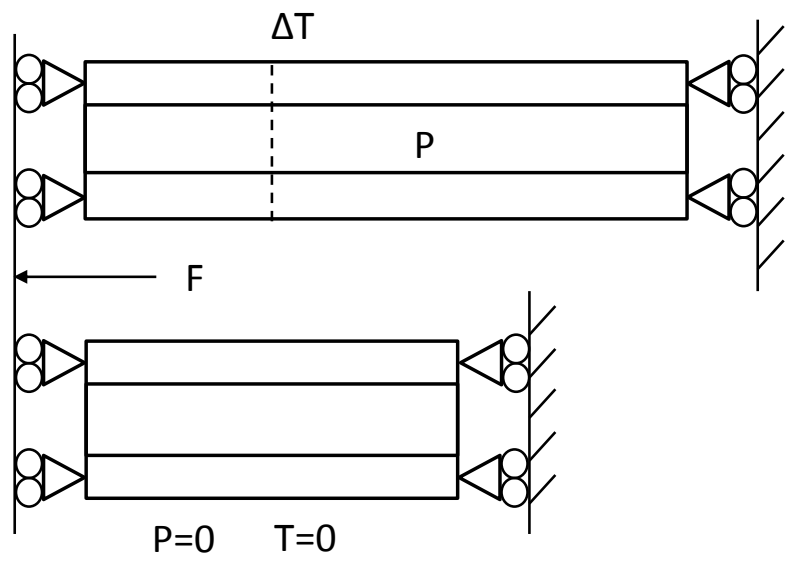

FIG. 4: PRESSURIZED TWO BAR 


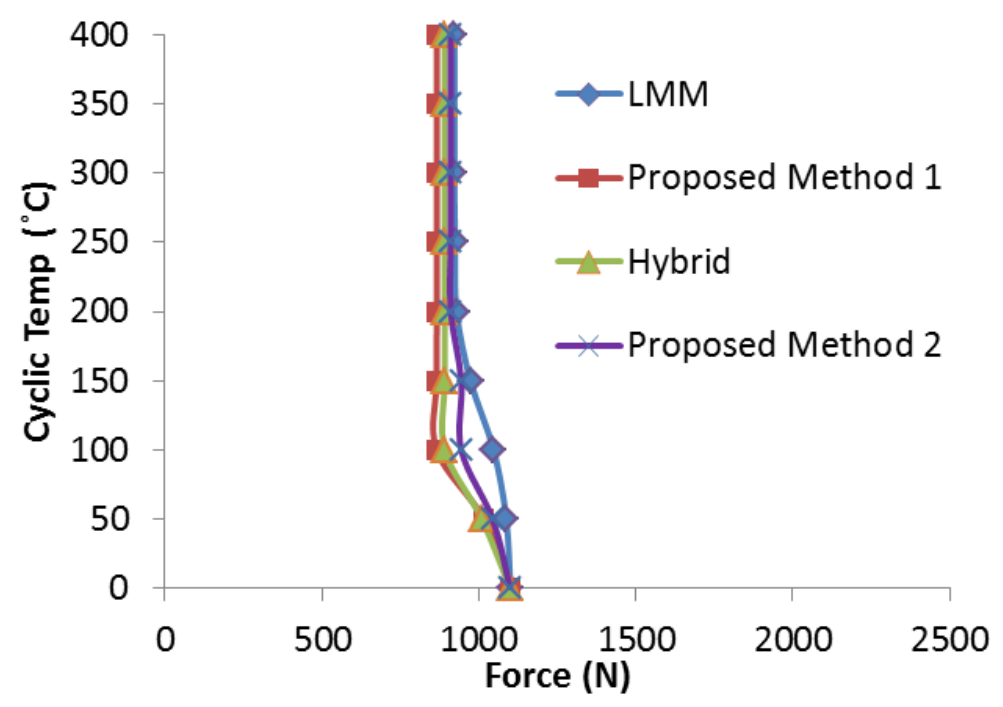

FIG. 5: RATCHET BOUNDARY $F / P=10$ 


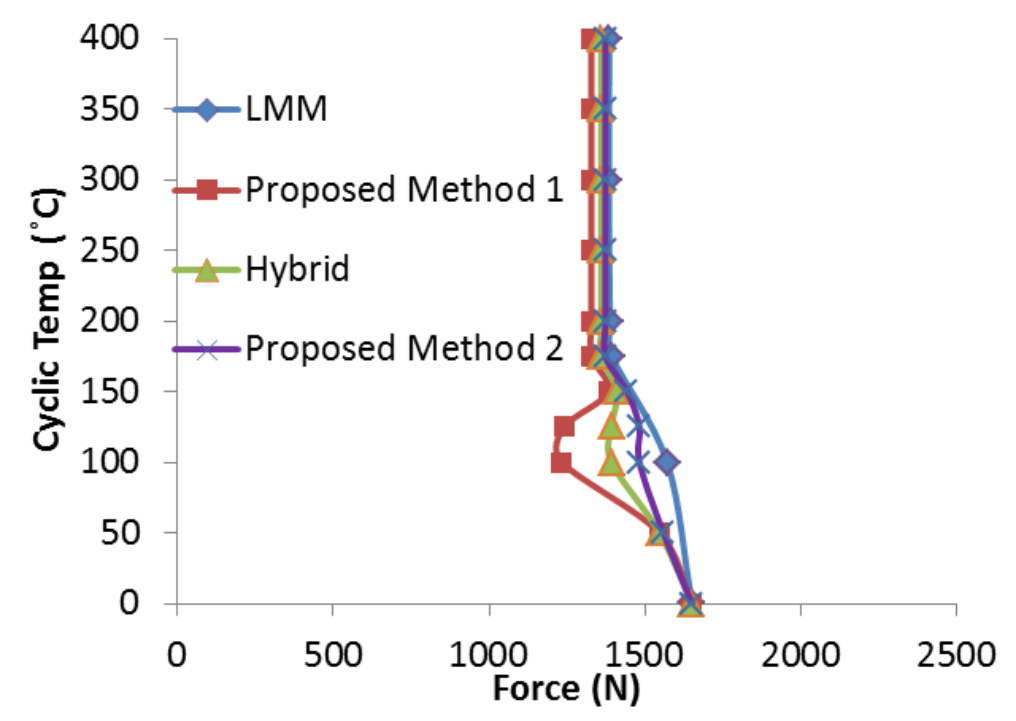

FIG. 6: RACHET BOUNDARY F/P=15 


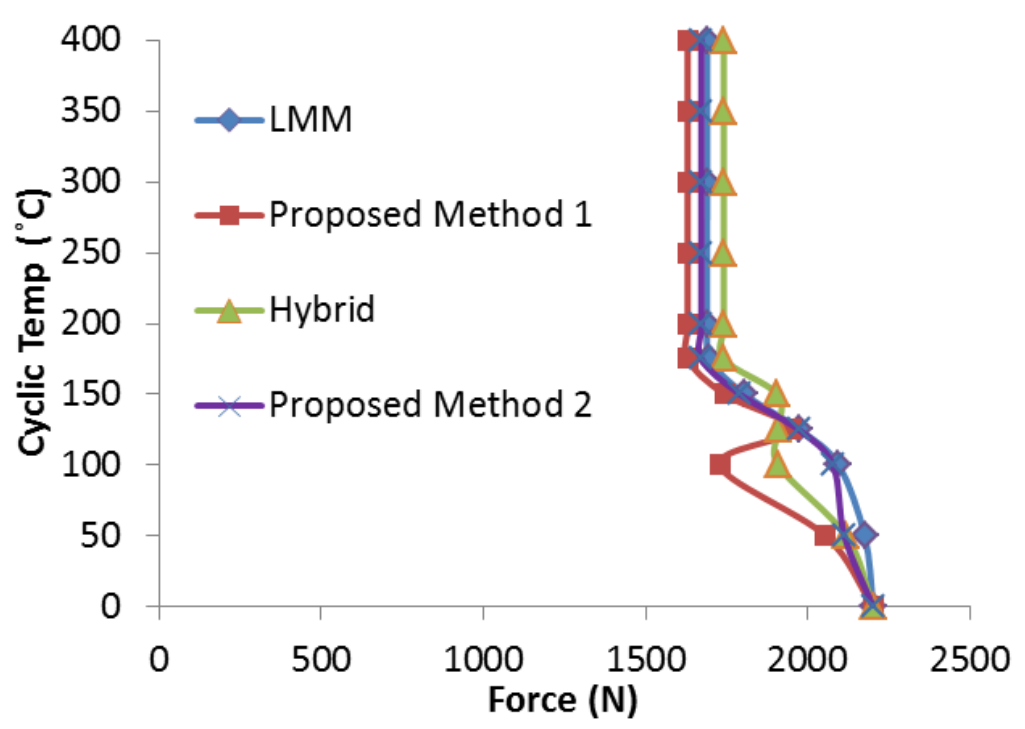

FIG. 7: RATCHET BOUNDARY F/P=20 

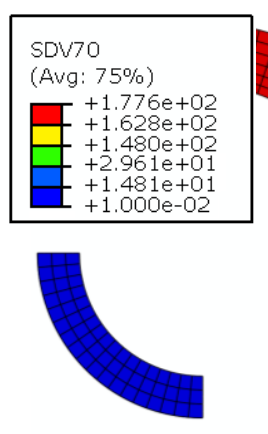
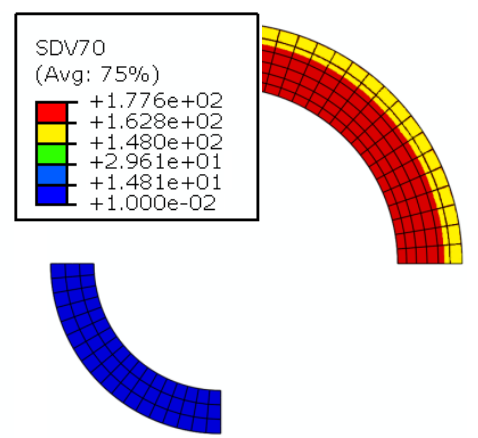

FIG. 8: MODIFIED YIELD STRENGTH $F / P=20 \Delta \theta=150^{\circ} \mathrm{C}$ LEFT: HYBRID, RIGHT: PROPOSED METHODS 

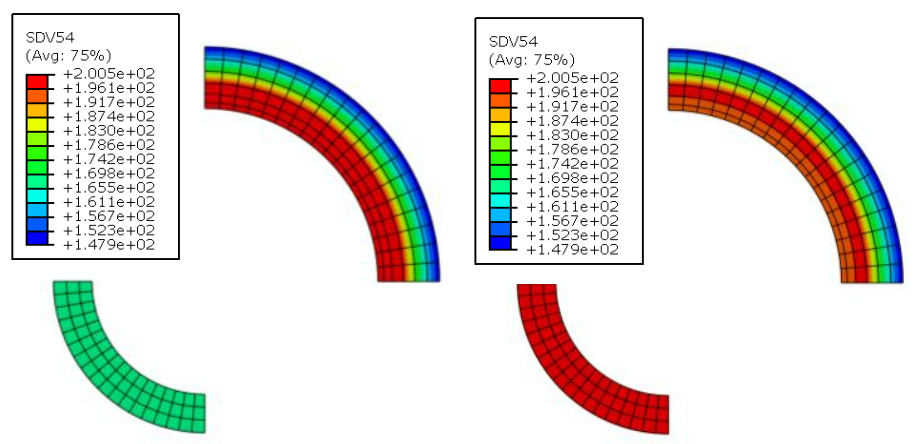

FIG. 9: EQUIVALENT TOTAL STRESS $F / P=15$

Left: $\Delta \theta=0^{\circ} \mathrm{C}$, Right: $\Delta \theta=150^{\circ} \mathrm{C}$ 